\title{
Acute Aortic and Mitral Valve Insufficiency Precipitated by Infective Endocarditis
}

\author{
Raj Patel, MD, Tatiana Bekker, MD, and Daniel Kramer, MD
}

\section{INTRODUCTION}

Acute severe valvular regurgitation is a medical emergency requiring prompt recognition and diagnosis. Both mitral and aortic valve rupture, if left untreated, inevitably lead to advanced heart failure, cardiovascular collapse, and death. As such, rapid diagnosis is critical and surgical valve repair or replacement is a life-saving intervention. We present a case of a patient with both mitral valve and aortic valve bacterial endocarditis who developed acute valvular rupture, regurgitation, and cardiovascular collapse.

\section{CASE PRESENTATION}

A 63-year-old male with no prior medical history presented to an outside hospital with shortness of breath, fatigue, and unexplained 30 pound weight loss over three months. He noted some chills, but otherwise denied fevers, night sweats, chest pain, or palpitations.

On admission to the outside facility, he was noted to be hypoxic and admitted to the intensive care unit (ICU) for respiratory failure. Chest computed tomography (CT) was unrevealing. There was concern for a non-ST elevation myocardial infarction (NSTEMI) for which a cardiac catheterization was performed. No coronary disease was noted on that study; however, it did reveal severe aortic insufficiency and severe mitral regurgitation with an ejection fraction of roughly $60 \%$. Blood cultures from admission showed Gram positive cocci in chains. No vegetations were noted on transthoracic echocardiogram (TTE) or subsequent transesophageal echocardiogram (TEE). The patient was transferred to Thomas Jefferson University Hospital (TJUH) for further management of his valvular disease.

On presentation to TJUH, his physical examination was notable for the following: thin male in mild respiratory distress, without jugular venous distension. Rales were present bilaterally on auscultation of the lungs. Cardiac findings included tachycardia with a grade II/VI diastolic decrescendo murmur heard at Erb's point as well as a grade III/VI holosystolic murmur at the cardiac apex radiating to the axilla. A repeat TTE was done showing a calcified mobile echodensity on the mitral valve with mitral insufficiency and a mobile calcified echodensity on the aortic valve with a flail leaflet and moderate to severe aortic insufficiency. His admission chest x-ray showed radiographic evidence of pulmonary edema. Blood cultures from the outside hospital grew Streptococcus gallolyticus subsp. pasteurianus (formerly Strep. bovis). Repeat blood cultures at TJUH were without growth, but the patient had been on antibiotics.

He was initially treated with diuretics for his edema, but remained in mild respiratory distress. As such, he was transferred to the cardiac ICU on hospital day 1 for closer monitoring. As he was relatively hemodynamically stable, appropriate preoperative work up was started and he was planned for non-emergent cardiac surgery on hospital day 8. On hospital day 5 however, his respiratory status rapidly worsened. He initially improved with bilevel positive airway pressure, but eventually decompensated, requiring intubation. Immediately after intubation, he became hypotensive, necessitating pressors. His post intubation chest radiograph was notable for acute pulmonary edema.

\section{OUTCOME AND FOLLOW-UP}

Cardiothoracic surgery was called and the patient was taken for emergency cardiac surgery. Intraoperatively, the right leaflet of the aortic valve was noted to be prolapsed and perforated. This was ultimately excised. The mitral valve was noted to have endocardial lesions involving the coapting surfaces of both anterior and posterior leaflets, and was also excised. Tissue valves were implanted to replace the damaged aortic and mitral valves. Operating room cultures of valvular tissue showed Gram positive cocci on staining, but the organism was deemed non-viable on repeat subculture.

Post-operatively the patient completed a 4-week course of intravenous (IV) antibiotics. His course was complicated by renal failure requiring dialysis and ventilator dependent respiratory failure requiring tracheostomy and percutaneous endoscopic gastrostomy (PEG) tube placement. Most recently he was seen as an outpatient and was doing well from a cardiac standpoint. He was no longer on dialysis and both tracheostomy and PEG tube had been removed. He was noted to be gaining weight and was able to walk multiple blocks without becoming short of breath. 


\section{DISCUSSION}

The primary mechanisms by which acute aortic regurgitation develops are endocarditis, aortic dissection, and trauma. Acute regurgitation presents as a distinct entity, quite different than the chronic form when the ventricle has time to compensate for increased volume. In acute aortic regurgitation there is a large reflux of volume into an unprepared left ventricle. This reflux of volume into a relatively small left ventricle rapidly increases the left ventricular end diastolic pressure. Eventually this pressure is transmitted to the left atrium and pulmonary circulation, precipitating pulmonary edema. Additionally, there is decreased cardiac output, which is exacerbated by two mechanisms. The rise in end diastolic pressure mediates a premature closure of the mitral valve thus shortening diastolic filling time. Furthermore, the drop in cardiac output will lead to a compensatory tachycardia that will also decrease diastolic filling time. ${ }^{1}$

Patients typically present with signs of acute cardiovascular collapse (profound hypotension, pallor, cool extremities) and acute pulmonary edema. Of note, due to the acute volume and pressure overload, a functional mitral regurgitation murmur may develop in the setting of acute aortic regurgitation. However, this is different from an organic cause of mitral regurgitation. It does not warrant any intervention on the mitral valve and should improve with repair of the aortic valve.1-3

Organic causes of acute mitral regurgitation, such as endocarditis, do however require rapid intervention to correct the valvular defect. Acute mitral valve rupture leads to an acute regurgitation of fluid into a normal sized, relatively small left atrium. This leads to acute pulmonary edema and decreased forward flow. ${ }^{1-3}$

Definitive treatment of acute valvular insufficiency is surgical repair. Temporizing medical measures can be of limited benefit-primarily intravenous vasodilators, such as nitroprusside, which promote forward flow by reducing afterload. ${ }^{3}$ Inotropic agents such as dobutamine can help as well.
The most common cause of acute valvular regurgitation is endocarditis. Some studies show that it accounts for $>56 \%$ of cases of acute aortic regurgitation. ${ }^{2}$ However, encountering multi-valvular disease is overall less common and has been estimated to account for only $15 \%$ of all cases of all infective endocarditis. ${ }^{4}$ The proposed mechanisms theorize that there is secondary infection of the mitral valve from either direct extension of the aortic valve infection or a jet of regurgitant infected material that progresses to a satellite infection. ${ }^{5}$ Cases of multi-valvular endocarditis tend to be more complex and the majority $(\sim 70 \%)$ require surgical intervention. ${ }^{4}$

\section{KEY POINTS}

Our patient presented with subacute, multivalvular endocarditis with evidence of valvular insufficiency on presentation. He initially appeared well compensated without significant respiratory compromise. However, the fragility of his valves placed him in a high-risk position. Upon acute valvular rupture he rapidly decompensated and required emergency cardiac surgery. Acute valvular insufficiency is a medical emergency. Rapid identification and triage, with referral for surgery, is a life-saving intervention.

\section{REFERENCES}

1. Hamirani YS, Dietl CA, Voyles W, Peralta M, Begay D, Raizada V. Acute aortic regurgitation. Circulation 2012;126:1121-1126.

2. Roberts WC, Ko JM, Moore TR, Jones WH. Causes of pure aortic regurgitation in patients having isolated aortic valve replacement at a single US tertiary hospital (1993 to 2005). Circulation 2006;114:422-429.

3. Mokadam NA, Stout KK, Verrier ED. Management of acute regurgitation in left-sided cardiac valves. Texas Heart Institute Journal 2011;38(1):9-19.

4. Selton-Suty C, Doco-Lecompte T, Bernard Y, et al. Clinical and microbiologic features of multivalvular endocarditis. Current infectious disease reports 2010;12(4):237-243.

5. Karalis DG, Bansal RC, Hauck AJ, et al. Transesophageal echocardiographic recognition of subaortic complications in aortic valve endocarditis. Circulation 1992:86(2):353-362 